\title{
Recapitulating nature's design: Myocardial repair with cell sheet technology
}

\author{
Leora B. Balsam, MD
}

\author{
From the Department of Cardiothoracic Surgery, New York University-Langone Medical Center, New York, NY. \\ Disclosures: Author has nothing to disclose with regard to commercial support. \\ Received for publication April 25, 2017; accepted for publication May 9, 2017; available ahead of print June 16, \\ 2017. \\ Address for reprints: Leora B. Balsam, MD, Department of Cardiothoracic Surgery, NYU-Langone Medical Cen- \\ ter, 530 First Ave, Suite 9V, New York, NY 10016 (E-mail: leora.balsam@nyumc.org). \\ J Thorac Cardiovasc Surg 2017;154:951-2 \\ 0022-5223/\$36.00 \\ Copyright (C) 2017 by The American Association for Thoracic Surgery \\ http://dx.doi.org/10.1016/j.jtcvs.2017.05.011
}

How close are scientists to engineering the adult human heart? The effort to repair and regenerate adult myocardium has moved from science fiction toward reality in the last two decades. Much of the focus has been on stem cells, basic cellular building blocks with the capability of proliferation, and differentiation into mature cell phenotypes. In the adult heart, a variety of progenitor cells have been identified, including resident cardiac stem cells and endothelial progenitor cells (EPCs), but in general their numbers are few and their ability to repair the heart after major injury is limited. Cell-based therapy has been proposed as a strategy to increase the local delivery of reparative cells to the injured area. The implanted cells might lead to myocardial repair through cardiomyocyte regeneration, angiogenesis, paracrine delivery of growth factors, or some combination of the three. A variety of stem cell sources have been evaluated, including embryonic stem cells, bone marrow stem cells (hematopoietic and mesenchymal), and, more recently, induced pluripotent stem cells. The latter two are more attractive as potential autologous sources for myocardial repair in the adult. Delivery methods have included local injection into the peri-infarct border zone, intracoronary infusion, and topical placement as a cell sheet in the area of injury.

In this issue of the Journal, Shudo and colleagues ${ }^{1}$ build on prior work from their laboratory in myocardial repair after infarction. This group previously described the development of a scaffold-free cell sheet of layered smooth muscle cells (SMCs) and bone marrow-derived EPCs for myocardial repair. ${ }^{2}$ The bilayer sheet is grown on a temperature-responsive polymer that enables adherence to the culture dish at normothermia and release at room temperature. Because enzymatic digestion is not needed to remove the cell sheet from the culture dish, cell-cell junctions and the basal extracellular matrix are preserved. Shudo and colleagues ${ }^{1}$ have found that this not only makes the cell sheets easier to manipulate but also improves their engraftment to injured myocardium when compared with

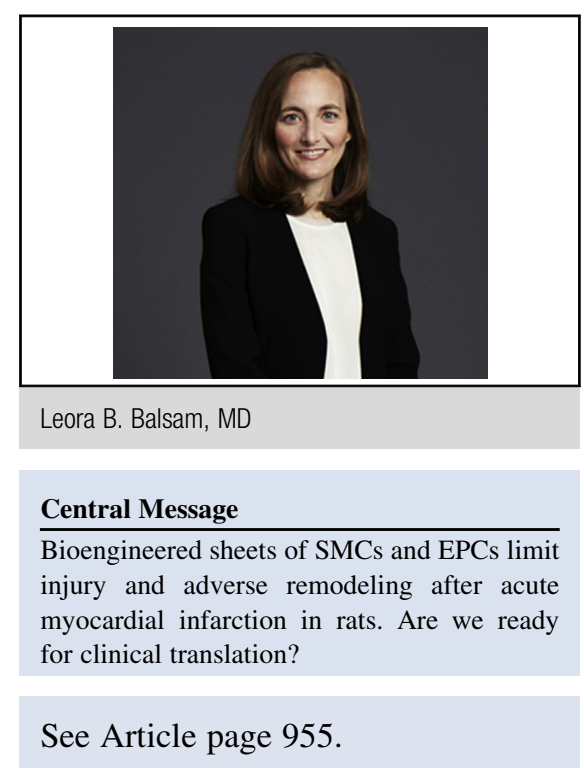

cell injections. In a rat model of acute myocardial infarction, they reported that cell sheets could be applied to the epicardium of the infarct zone, and 4 weeks later, capillary density in the border-zone myocardium was increased when compared with local injection of EPCs and SMCs in suspension. In addition, cell sheet-derived SMCs and EPCs were identified in mature vascular structures, confirming the direct involvement of donor cells from the cell sheet in angiogenesis. In the current study, Shudo and colleagues ${ }^{1}$ again create a bilayer cell sheet of SMCs and EPCs; however, the source of SMCs is different. Unlike in the prior study, in which SMCs were cultivated from thoracic aortic tissue, in the current study they are derived from bone marrow mesenchymal stem cells (MSCs). By culturing MSCs on fibronectin-coated plates, the cells are directed toward an SMC lineage. 1,3 Shudo and colleagues ${ }^{1}$ suggest that using MSCs (rather than aorta) as the source for SMCs is a more readily translatable clinical strategy.

As in their prior study, Shudo and colleagues ${ }^{1}$ evaluate the bone marrow-derived cell sheet in a rat model of acute myocardial infarction. Four weeks after treatment, there is a trend toward increased capillary density with cell sheet treatment compared with no treatment $(P=.08)$. Left ventricular function is significantly better and left ventricular dilatation is less after cell sheet treatment. Shudo and colleagues ${ }^{1}$ conclude that the novel cell sheet, derived entirely from bone marrow cells, augments 
neovascularization and limits adverse remodeling after myocardial infarction. The evidence for neovascularization is not as strong as in their prior study, ${ }^{2}$ perhaps because of the small sample size, and cell-fate tracking studies were not performed. Without these, the mechanistic basis underlying the effect on post-infarct remodeling remains unknown.

Cell-based therapies have quickly jumped from the bench to bedside. A variety of cell types, cell concentrations, and delivery methods have been used to treat patients with acute myocardial infarction and chronic ischemic heart disease in more than 40 randomized clinical trials. ${ }^{4,5}$ The results have been disappointing, which suggests that the therapy does not work or the conditions needed for it to succeed have not been identified. Is cell sheet technology a better way to go? Perhaps, but there are several obstacles that stand between the cell sheets described by Shudo and colleagues $^{1}$ and their clinical implementation. First, the cell sheets are generated after several weeks of culture; this is obviously not practical for treatment of acute myocardial injury, and whether it will be of benefit in the chronic phase of ischemic cardiomyopathy requires separate investigation. Second, the advantage of the bilayer construct is uncertain; side-by-side comparative testing with EPC-only cell sheets and SMC-only cell sheets should be performed. Third, although the investigators are able to coax MSCs toward a SMC phenotype by growing them on fibronectin-coated plates, the resulting cell population is far from pure. When MSCs were cultured under these conditions, only $65 \%$ of cells expressed the SMC marker alpha-SMC actin, whereas $57 \%$ of cells still expressed the MSC marker CD105. ${ }^{3}$ Off-target effects of introducing a mixed population of cells, that is, MSCs as well as the intended SMCs and EPCs, into the peri-infarct milieu require further investigation. Last, this preclinical study was performed in young previously healthy animals. Existing science suggests that the efficacy of cell sheets derived from older donors with cardiovascular comorbidities will be worse.

\section{References}

1. Shudo Y, Goldstone AB, Cohen JE, Patel JB, Hopkins MS, Steele AN, et al Layered smooth muscle cell-endothelial progenitor cell sheets derived from the bone marrow augment post-infarction ventricular function. J Thorac Cardiovasc Surg. 2017;154:955-63.

2. Shudo Y, Cohen JE, MacArthur JW, Atluri P, Hsiao PF, Yang EC, et al. Spatially oriented, temporally sequential smooth muscle cell-endothelial progenitor cell bi-level cell sheet neovascularizes ischemic myocardium. Circulation. 2013; 128(Suppl 1):S59-68.

3. Shudo Y, Cohen JE, Goldstone AB, MacArthur JW, Patel J, Edwards BB, et al. Isolation and trans-differentiation of mesenchymal stromal cells into smooth muscle cells: utility and applicability for cell-sheet engineering. Cytotherapy. 2016;18:510-7

4. Fisher SA, Zhang H, Doree C, Mathur A, Martin-Rendon E. Stem cell therapy for acute myocardial infarction. Cochrane Database Syst Rev 2015;9: CD006536.

5. Fisher SA, Doree C, Mathur A, Taggart DP, Martin-Rendon E. Stem cell therapy for chronic ischaemic heart disease and congestive heart failure. Cochrane Database Syst Rev 2016;12:CD007888. 